\title{
Recurrent abdominal pain in children: a clinical approach
}

Seng Hock Quak, MBBS, MMed

ABSTRACT The term 'recurrent abdominal pain', or RAP, refers mainly to the duration of painful period and frequency of pain. The commonly accepted duration is at least three months in the preceding period, and over this three-month period, there are at least three episodes of pain that are severe enough to affect the daily activities of the affected patients. Over the years, with advances in medical technology and better understanding of the pathophysiology of abdominal pain, more and more organic causes have been identified. However, the most common cause of RAP in children is still functional in origin.

Keywords: carbohydrate intolerance, eosinophilic oesophagitis, surgical abdomen

\section{INTRODUCTION}

Abdominal pain is one of the most common symptoms in children. Often, the pain is of acute onset and may be due to a number of gastrointestinal (GI) or extraintestinal causes. These include infection of the Gl tract, dietary indiscretion, urinary tract infection, and other more sinister surgical conditions such as acute appendicitis. With the appropriate management, such acute abdominal pain usually does not lead to untoward long-term sequelae. However, there are a number of difficulties in the management of children with abdominal pain. First, in young children, the history of pain is often 'second-hand', as it is the parent's or caregiver's interpretation of the child's reaction to some abdominal upset. Despite this difficulty, most parents are able to offer very useful leads to the aetiology of abdominal pain in their children. Furthermore, abdominal examination of young children with abdominal pain is challenging.

Recurrent abdominal pain (RAP) is common in children, and affects about $10 \%-20 \%$ of school-going children. ${ }^{(1)}$ Apley and Naish, ${ }^{(2)}$ who documented RAP in children in the middle of last century, noted that in the vast majority of cases, no organic causes could be found, and they considered the aetiology of RAP to be psychogenic in origin. In young children, the localisation of abdominal pain is often vague, and frequently, patients would indicate that the pain is located in the central region of the abdomen. The severity and frequency of pain bear no relation to its aetiology. ${ }^{(2)}$

The term 'RAP' refers mainly to the duration of painful period and frequency of pain. The commonly accepted duration is at least three months in the preceding period, and over this three-month period, there are at least three episodes of pain that are severe enough to affect the daily activities of the affected patients. Over the years, with advances in medical technology and better understanding of the pathophysiology of abdominal pain, more and more organic causes have been identified. However, the most common cause of RAP in children is still functional in origin.

\section{ORGANIC CAUSES OF RAP Helicobacter pylori infection}

In recent years, Helicobacter pylori (H. pylori) infection has been known to cause gastroduodenal disease. Since its identification in the 1980s, H. pylori has been the subject of many studies performed to ascertain whether the bacterium is the cause of RAP in children. Although the prevalence rate of $H$. pylori infection in children with duodenal ulcer is high, its prevalence rate among children with RAP is inconsistent. Many children suffering from RAP with associated $H$. pylori do not benefit from eradication of the infection, as the pain persists even after successful treatment. On the other hand, many children in developing countries who have $H$. pylori in their gastric mucosa are completely asymptomatic. Hence, the role of H. pylori in RAP in children is far from clear. Macarthur et $\mathrm{al}^{(3)}$ reviewed 45 studies of children with RAP and concluded that $H$. pylori is unlikely to be the sole infection aetiological agent in this age group.

The diagnosis of $H$. pylori should be properly made with positive urea breath test, rapid urease test or histology. Serology alone is inadequate for making the diagnosis of active H. pylori infection, as it is only a marker of past exposure and should not be used as a diagnostic test.

\section{Eosinophilic oesophagitis and reflux oesophagitis}

Oesophagitis is a well-established cause of epigastric pain in children. Gastroesophageal reflux (GER) is common in young infants, and in the vast majority of cases, the reflux symptoms improve when the infants assume an erect posture and on introduction of solid food. Hence, symptoms of GER persist beyond the first two years of life in only a minority. ${ }^{(4)}$ In older children, GER may be precipitated by overeating, obesity and a sedentary lifestyle.

In recent years, eosinophilic oesophagitis (EE) has been identified with increasing frequency in Western countries and developing countries. ${ }^{(5,6)}$ The presenting features are almost identical to those of GER and the only way to make a diagnosis of $\mathrm{EE}$ is by mucosal biopsies. ${ }^{(7,8)}$ Often, there is an increase in 
eosinophils in the mucosal or submucosal regions, and the number of eosinophils consistent with the diagnosis of EE is 15 or more per high power field. In severe cases, this increase in eosinophils can be seen in not only the oesophagus, but also the stomach and small and large intestines. When the disease is present for a long period of time, the symptoms become more severe, with increasing fibrosis of the affected oesophagus. Obstructive symptoms such as dysphagia and food impaction can also occur.

Allergy is considered to be the aetiology of EE. However, the specific allergen is frequently not identified. In young children, food items such as milk, soy, egg and fish protein are at the top of the list of suspected allergens..$^{(9)}$ As mentioned earlier, the diagnosis of EE is by histology, and thus, testing for food antibody or skin prick test with specific food items are not useful in determining the presence or absence of EE. Treatment is topical corticosteroids. Obviously, the offending food item should be avoided. ${ }^{(9)}$ In severe cases, oesophageal dilatation may be necessary.

\section{Carbohydrate intolerance}

In Asia, carbohydrate intolerance is a common cause of RAP. ${ }^{(10,11)}$ The most common carbohydrate is lactose, which is found in mammalian milk. The onset of lactose intolerance is insidious and the affected patients may not realise that lactose is the culprit of their abdominal pain, as the pain only appears a few hours after milk ingestion. It is associated with increase in flatulence, with or without diarrhoea. Other carbohydrates that may be responsible for RAP include sorbitol, a food additive.

Clinical diagnosis of carbohydrate intolerance can be made by means of food withdrawal and challenge. When the suspected carbohydrate is omitted from the diet, the abdominal pain should improve. With the reintroduction of the same carbohydrate in the diet, abdominal pain should recur. Laboratory tests, such as breath hydrogen test, are often unnecessary.

\section{Abdominal migraine}

RAP can be a manifestation of migraine in children. Often, there is a positive family history and the abdominal pain is usually associated with nausea or vomiting. Headache may or may not be present. Abdominal migraine seldom persists into adulthood. However, evidence suggests that it is a precursor of migraine headache.

\section{Inflammatory bowel disease}

Chronic inflammatory bowel disease (IBD) is often suspected in children with RAP. The diagnosis of IBD is often delayed, as the presenting features are nonspecific. Vague abdominal pain is frequently the only symptom. In Asia, the incidence of IBD is increasing, and this phenomenon may be due to a number of reasons. The most common postulation is the hygiene hypothesis. Over the years, as our surrounding transforms from an agricultural society to a clean and developed industrialised environment, the lack of common infections and parasites in our intestinal tracts has been postulated as a possible aetiology for the development of IBD. ${ }^{(12-14)}$ Meanwhile, changes in our diet, such as the increased consumption of highly refined food and animal fat, coupled with low intake of fruit and vegetables, could also be possible factors. Food additives found in processed food and pesticides may play a role in the increasing frequency of IBD among many emerging economies.

Other than abdominal pain, other associated features include loss of appetite, general lethargy, fever, diarrhoea and nonintestinal manifestations such as arthralgia, arthritis or delay in pubertal developments. IBD should be suspected if a patient with RAP also has either poor weight gain or weight loss.

\section{Surgical conditions}

A number of surgical conditions can present as RAP. These include recurrent intussusceptions, Meckel diverticulitis, intestinal malrotation, choledochal cyst and intestinal lymphoma. Diagnosis of recurrent intussusceptions is challenging. Patients should be examined when they are in pain, as in between the painful periods, the abdominal examination can be normal. It would also be most helpful to perform abdominal ultrasonography when the patient is experiencing pain. Meckel diverticulum is a common intestinal malformation that is present in about $2 \%$ of the population. It can present in various ways, including RAP and intestinal haemorrhage, and can also be the lead point for intussusceptions. When it presents as abdominal pain, ${ }^{(15,16)}$ inflammation or ulceration of the ectopic gastric mucosa is the usual cause. Pain is often localised the right iliac fossa. The diagnosis can be confirmed by performing a radioactive Meckel scan. The radioactive tracer is picked up by the ectopic gastric mucosa and would appear as bright spots at the right iliac fossa region. Treatment is surgical resection of the diverticulum.

Intestinal malrotation is a surgical emergency if it presents as abdominal pain and vomiting. It is not uncommon for such cases to be diagnosed as gastritis or gastric flu and the patient is prescribed anti-emetics. Pain and vomiting indicate that the small intestine has undergone volvulus. ${ }^{(17-19)}$ Volvulus can undo itself and present as RAP. The hallmark of malrotation is typically the heavily bile-stained vomitus. The colour of the vomitus appears bright green. Surgical correction should be performed at diagnosis, as the patient is at risk of losing the whole midgut, a part that is essential for digestion and nutrient absorption.

Choledochal cyst is more common among Asian girls and may present in a classical triad - RAP, recurrent jaundice and a palpable mass at the right hypochondrium. However, the occurrence of this triad is not common. Jaundice may not be present, and the cyst may be small and not easily palpable. Choledochal cyst should be suspected when pain or tenderness is located at the right hypochondrium. The diagnosis can be confirmed by performing an abdominal ultrasonography. Intestinal lymphoma is one of the most sinister causes of RAP in children. The pain can be persistent for some period before obstructive symptoms occur. One should suspect this condition if the child with RAP also has poor food intake and weight loss. Pain may be aggravated by food intake. Gynaecological conditions ${ }^{(20,21)}$ in young children that may present as RAP include ovarian 


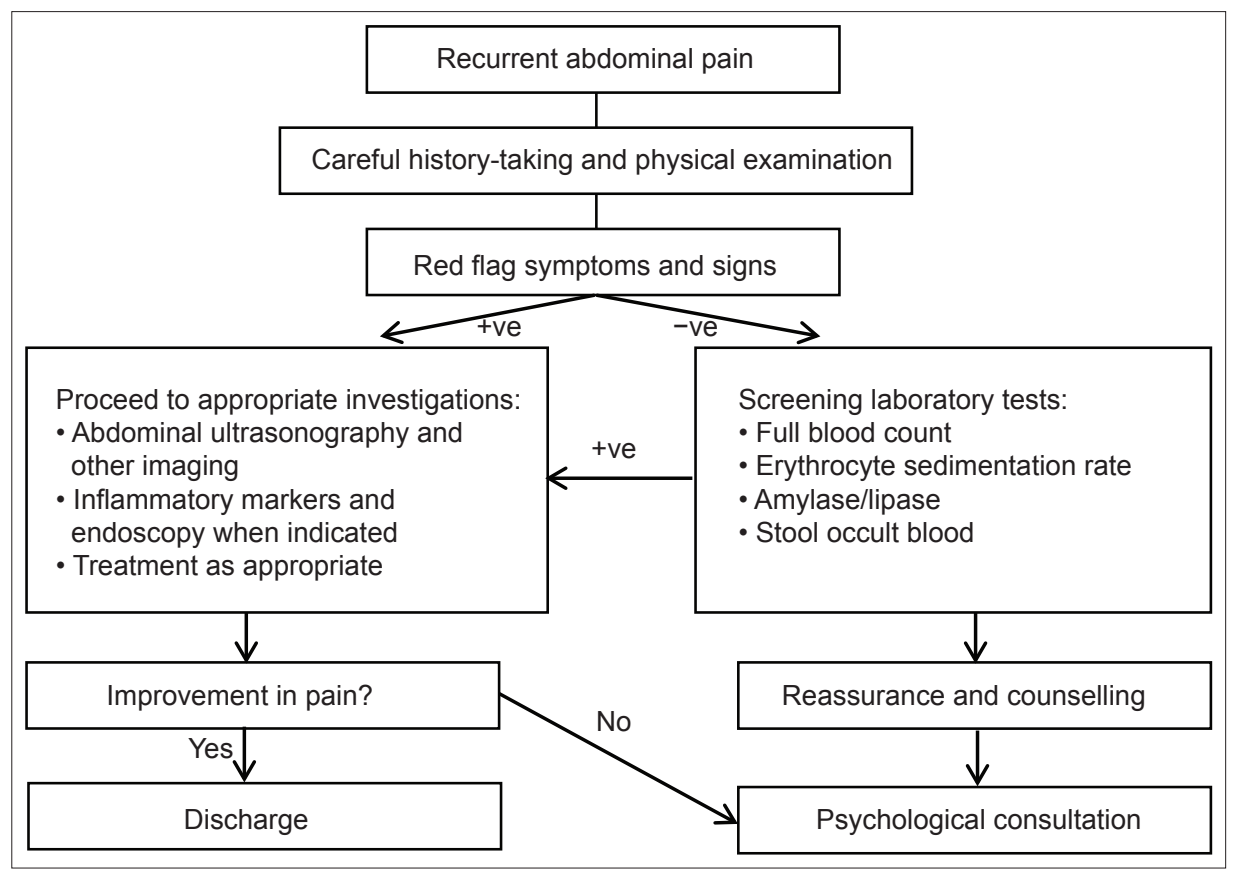

Fig. 1 Algorithm for children with recurrent abdominal pain.

cyst, teratoma and dysmenorrhoea. These conditions should be considered if the pain is located more in the lower abdomen.

\section{CLINICAL APPROACH}

When a young child is seen for RAP, the most important approach to take is detailed history-taking and careful clinical examination (Fig. 1). Other than looking for causes related to the Gl causes, extraintestinal aetiologies such as genitourinary causes should also be excluded. The common red flag symptoms of RAP and red flag signs of RAP in children are listed in Tables I and II, respectively.

When performing a physical examination, particular attention should be paid to excluding the organic causes mentioned earlier. There are a number of red flag signs that indicate an organic aetiology. When good clinical history and physical examination are unable to define a specific aetiology, some screening laboratory tests, such as erythrocyte sedimentation rate, stool for occult blood and urinalysis, may be performed. In cases where no obvious organic causes are found, both the child and the parents should be reassured and counselled. As the patient's health-related quality of life may be affected, ${ }^{(22,23}$ psychological consultions are often necessary. In other words, in the absence of clinical, laboratory and radiological abnormalities, the cause of RAP is classified as functional and considered a form of functional Gl disorder.

The main aim of management of children with RAP is to teach the child to cope with the pain and to improve the child's quality of life. A multidisciplinary team approach is the most ideal in dealing with this type of complex problem. Medical treatment with Gl prokinetic or antispasmodic medications has been proven to be disappointing. Both the child and the parents should be counselled on stress coping strategies and provided with ample reassurance that there is no serious organic disease.
Table I. Red flag symptoms of recurrent abdominal pain (RAP).

\begin{tabular}{l} 
Red flag symptoms \\
\hline - Localisation of pain away from the central abdominal region \\
- Pain associated with changes in bowel habit, particularly \\
diarrhoea, constipation or nocturnal bowel movement \\
- Pain associated with night-waking \\
- Constitutional symptoms such as recurrent fever, and loss of \\
appetite and energy \\
- RAP in very young children aged < 4 years \\
- History of weight loss or poor growth \\
- Recurrent unexplained fever \\
- Recurrent bloody stools \\
- Unexplained pallor
\end{tabular}

Table II. Red flag signs of recurrent abdominal pain in children.

\section{Red flag signs}

- Evidence of weight loss or decline in height velocity

- Pallor/jaundice

- Organomegaly or palpable mass in the abdomen

- Localised tenderness away from the umbilical region

- Perianal abnormalities

- Joint swelling, redness or warmth, finger clubbing

- Ventral hernia of the abdominal wall

Modifications of lifestyle may be helpful. Dietary intervention is an important strategy adopted by many parents in managing RAP in their children. Increasing the amount of daily fibre intake has marginal effect in improving abdominal pain. The use of probiotics has not been shown to be useful. Behavioural therapy for the management of children with RAP has been practised in many centres in the world. Compared with standard therapies, cognitive behavioural therapy may be a more useful intervention for children with RAP, ${ }^{(24,25)}$ although evidence for its benefit remains weak and further large-scale multicentre studies are required to confirm its usefulness. 


\section{REFERENCES}

1. Oh MC, Aw MM, Chan YH, Tan LZ, Quak SH. Epidemiology of recurrent abdominal pain among Singapore adolescents. Ann Acad Med Singapore 2004; 33(5 Suppl):S10-1.

2. Apley J, Naish N. Recurrent abdominal pains: a field of 1,000 school children. Arch Dis Child 1958; 33:165-70.

3. Macarthur C, Saunders N, Feldman W. Helicobacter pylori, gastroduodenal disease, and recurrent abdominal pain in children. JAMA 1995; 273:729-34.

4. Vandenplas Y, Rudolph CD, Di Lorenzo C, et al. Pediatric gastroesophageal reflux clinical practice guidelines: joint recommendations of the North American Society for Pediatric Gastroenterology, Hepatology, and Nutrition (NASPGHAN) and European Society for Pediatric Gastroenterology, Hepatology, and Nutrition (ESPGHAN). J Pediatr Gastroenterol Nutr 2009; 49:498-547.

5. Tan MLN, Wong S, Srivastava S, et al. Esinophilic esophagitis in a predominantly Chinese population of children is uncommon. 4th World Congress of Pediatric Gastroenterology, Hepatology and Nutrition 2012, Taiwan. (Abstract PP12022)

6. Furuta GT, Forbes D, Boey C, et al. Eosinophilic gastrointestinal disease (EGIDs). J Pediatr Gastroenterol Nutr 2008; 47:234-8.

7. Furuta GT, Liacouras CA, Collins $\mathrm{MH}$, et al. Eosinophilic esophagitis in children and adults: a systematic review and consensus recommendations for diagnosis and treatment. Gastroenterology 2007; 133:1342-63.

8. Dellon ES, Gibbs WB, Fritchie KJ, et al. Clinical, endoscopic, and histologic findings distinguish eosinophilic esophagitis from gastroesophageal reflux disease. Clin Gastroenterol Hepatol 2009; 7:1305-13.

9. Spergel JM, Brown-Whitehorn TF, Cianferoni A, et al. Identification of causative foods in children with eosinophilic esophagitis treated with an elimination diet. J Allergy Clin Immunol 2012; 130:461-7.e5.

10. Boey CC. Lactase deficiency among Malaysian children with recurrent abdominal pain. J Paediatr Child Health 2001; 37:157-60.

11. Quak SH. Lactose intolerance in Singapore children. Asian Oceanian J Pediatr Child Health 2002; 2:85-98.

12. Quah SH. Role of probiotics and nutrition in the management of chronic inflammatory bowel disease in children. Singapore Med J 2013; 54:183-4.

13. Ponder A, Long MD. A clinical review of recent findings in the epidemiology of inflammatory bowel disease. Clin Epidemiol 2013; 5:237-47.

14. Weinstock JV, Elliott DE. Helminths and the IBD hygiene hypothesis. Inflamm Bowel Dis 2009; 15:128-33.

15. Huang CC, Lai MW, Hwang FM, et al. Diverse presentations in pediatric Meckel's diverticulum: a review of 100 cases. Pediatr Neonatal 2014; 55:369-75.

16. Rho JH, Kim JS, Kim SY, et al. Clinical Features of Symptomatic Meckel's Diverticulum in Children: Comparison of Scintigraphic and Nonscintigraphic Diagnosis. Pediatr Gastroenterol Hepatol Nutr 2013; 16:41-8.

17. Nehra D, Goldstein AM. Intestinal malrotation: varied clinical presentation from infancy through adulthood. Surgery 2011; 149:386-93.

18. Millar AJ, Rode H, Cywes S. Malrotation and volvulus in infancy and childhood. Semin Pediatr Surg 2003; 12:229-36.

19. Sheikh F, Balarajah V, Ayantunde AA. Recurrent intestinal volvulus in midgut malrotation causing acute bowel obstruction: A case report. World J Gastrointest Surg 2013; 27:43-6.

20. Mukhopadhyay M, Shukla RM, Mukhopadhyay B, et al. Ovarian cysts and tumors in infancy and childhood. J Indian Assoc Pediatr Surg 2013; 18:16-9.

21. Sasaki KJ, Miller CE. Adnexal torsion: review of the literature. J Minim Invasive Gynecol 2014; 21:196-202.

22. Petersen S, Hägglöf BL, Bergström El. Impaired health-related quality of life in children with recurrent pain. Pediatrics 2009; 124:e759-67.

23. Spuijbroek AT, Oostenbrink R, Landgraf JM, et al. Health-related quality of life in preschool children in five health conditions. Qual Life Res 2011; 20:779-86.

24. Rutten JM, Reitsma JB, Vlieger AM, Benninga MA. Gut-directed hypnotherapy for functional abdominal pain or irritable bowel syndrome in children: a systematic review. Arch Dis Child 2013; 98:252-7.

25. Huertas-Ceballos A, Logan S, Bennett C, Macarthur C. Psychosocial interventions for recurrent abdominal pain (RAP) and irritable bowel syndrome (IBS) in childhood. Cochrane Database Syst Rev 2008; 23:CD003014. 